Results Nearly all FM were female (98\%); main reason for exclusion was the inability to interrupt drugs with analgesic properties for $>10$ days. Age [FM mean 49(SEM1) vs C 46(2) y.], gender and socio-demographic characteristics were similar in both groups. Mean duration of symptoms was 8 y (range 0.449). RPS was 64(13-98), VAS was 5.7 (SD 2). Means for clinical examination were: TP 16(0.3), MS 29(0.9) and PGI 2.7(0.1). RIII reflex threshold was reduced in FM [26(2) vs 38(2) mA, p < 0.01]. Thermal perceptions (hot and cold) were similar in both groups, whereas thermal pain thresholds and latency (hot, cold, and cold pressure test) were drastically decreased in $\operatorname{FM}(\mathrm{p}<$ 0.001). Correlation analysis showed a significant relationship between PGI, and central QST(Pearson $r=-0.28 ; \mathrm{p}=0.02$ ) and between PGI, MS and $\mathrm{TP}(\mathrm{r}=0.34$ and $\mathrm{r}=0.66$, respectively $\mathrm{p}<0.01)$. Correlation with CYP2D6 activity is under analysis.

Conclusion Physician global impression, but not the other clinical scores, correlates with the extent of central sensitisation. Supported by the SNSF (3200-056028.98).

\section{SAT0131 INTERNAL IMAGE OF ILLNESS IN FIBROMYALGIA AND SYSTEMIC LUPUS ERYTHEMATOSUS}

AB Zborovsky, SA Kharchenko, GP Suleymanova, RA Grekhoff. Laboratory for Soft Tissue Rheumatism, Research Institute for Clinical and Experimental Rheumatology, Volgograd, Russia

\subsection{6/annrheumdis-2001.590}

Background The disturbances of the system of personality's attitudes and psychological specificity of the disease perception reflect in the patient's behaviour that plays an important role in therapy and rehabilitation.

Objectives The aim of present study was to investigate some elements of internal image of illness, including some aspects of psychological status in patients with fibromyalgia (FM) and systemic lupus erythematosus (SLE).

Methods Using a battery of psychological tests, we determined the level of neurotic disturbances, the level of subjective control (Health Locus of Control), the type of attitude towards illness (TOBOL) and the intensity of basic mechanisms of psychological defence (Life Style Index) in 32 patients with FM and 55 patients with SLE.

Results The results of our study are the evidence of considerable expressed anxiety and asthenia, low scores on general internality and internality concerning health and illness scales in FM and SSc patients.

The structure of internal image of illness represented by sensitive and ergopathic types in FM patients, and sensitive, ergopathic, hypochondriac and neurasthenic types of attitudes towards illness ? in SLE patients. We revealed a narrow spectrum of intense psychological defence: ?jet formation?, ?projection?, and ?regression? in FM patients, and ?jet formation?, ?projection?, and ?intellectualisation? ? in SLE patients.

A reliable differences were founded between FM and SLE patients: depression was more expressed in SLE than in FM patients $(\mathrm{p}<0,05)$, the index of internality concerning health and illness was higher in FM than in SLE patients $(p<0,05)$. Besides, the anxious and sensitive types of reacting towards illness are more frequent in FM than in SLE patients $(p<0,05)$. Conclusion The data we obtained indicate the development of substantial neurotic disturbances in FM and SLE patients. The decrease of subjective control?s level, especially in SLE patients are the evidence that these patients may be particularly likely to develop the belief that their disease is beyond their effective control. These patients, therefore, perceive that they will not be able to reduce substantially the pain, disabilities, or other sequel of the disease. It may lead to maladaptive behaviour such as nonadherence to treatment regimens, reliance upon ?quack? cures, and excessive use of medicines.

The mixed type of attitudes towards illness that we diagnosed in FM and especially in SLE patients indicate the discrepant nature of the structure of personal attitudes related to the disease. An essential pressure and prevalence of founded types of psychological defence indicate to narrowing of adaptive abilities of FM and SLE patients.

The results of research we carried out may be useful to correct nonadaptive attitudes of patients towards the disease in good time, to use psychological interventions in order to increase the efficiency of the therapy and to optimise rehabilitation.

\section{SAT0132 VALIDATION OF THE 'QUALITY OF LIFE SCALE' (QOLS-G) IN PATIENTS WITH FIBROMYALGIA}

${ }^{1} \mathrm{M}$ Offenbaecher, ${ }^{2} \mathrm{M}$ Waltz, ${ }^{1} \mathrm{G}$ Stucki. 'Physical Medicine and Rehabilitation, University Hospital, Munich; ${ }^{2}$ Rheumatology Service, St. Willibrord Hospital, Emmerich, Germany

\subsection{6/annrheumdis-2001.591}

Background The Quality of Life Scale (QOLS) is a 16 item questionnaire. The QOLS covers a broader perspective of quality of life as it is seen by patients with rheumatic diseases including non-health factors as satisfying relationships with others, material well being, self-fulfilment and leisure activities.

Objectives Our objective was to translate the QOLS into German and to evaluate its reliability and validity for the use of German speaking patients with fibromyalgia (FM).

Methods We administered the QOLS to 57 FM patients meeting the ACR criteria (14 patients filled out the questionnaire 1 week later) together with German versions of the FIQ, the SF-36 and a tender point count (TPC). All patients were asked about the severity of pain today (VAS) and the duration of symptoms; pressure pain thresholds (PPT) were assessed by dolorimetry at all tender points with a Fisher Dolorimeter and laboratory tests were obtained. Test-retest reliability was assessed using Spearman correlations. Internal consistency was evaluated with Cronbach's alpha of reliability. Construct validity of the QOLS was evaluated by correlating the QOLS with the FIQ and the SF-36 scores as well as with the pain variables and dolorimetry scores. Results Age range was 33-73 years (mean 53,8). Means were for the TPC (maximal achievable score 120 ) 67 , for pain today 6,8 and for duration of symptoms 11,8 years; the mean tenderness threshold was $1,49 \mathrm{~kg} / \mathrm{cm}^{2}$. Test-retest reliability was for the total QOLS 0,76. Internal consistency was 0,88. As hypothesised, low to moderate correlations (rho) were obtained between the QOLS and the total FIQ $(-0,29)$, the SF-36 (e.g. physical component 0,19 , mental component $-0,17$ ) as well as the pain variables (VAS - 0,18 , TPC -0,01) and PPT $(0,12)$.

Conclusion The QOLS-G is a reliable and valid instrument for measuring quality of life in German patients with FM. 